\title{
Ferrets as Models for Influenza Virus Transmission Studies and Pandemic Risk Assessments
}

\author{
Jessica A. Belser, Wendy Barclay, lan Barr, Ron A.M. Fouchier, Ryota Matsuyama, \\ Hiroshi Nishiura, Malik Peiris, Charles J. Russell, Kanta Subbarao, Huachen Zhu, Hui-Ling Yen
}

The ferret transmission model is extensively used to assess the pandemic potential of emerging influenza viruses, yet experimental conditions and reported results vary among laboratories. Such variation can be a critical consideration when contextualizing results from independent risk-assessment studies of novel and emerging influenza viruses. To streamline interpretation of data generated in different laboratories, we provide a consensus on experimental parameters that define risk-assessment experiments of influenza virus transmissibility, including disclosure of variables known or suspected to contribute to experimental variability in this model, and advocate adoption of more standardized practices. We also discuss current limitations of the ferret transmission model and highlight continued refinements and advances to this model ongoing in laboratories. Understanding, disclosing, and standardizing the critical parameters of ferret transmission studies will improve the comparability and reproducibility of pandemic influenza risk assessment and increase the statistical power and, perhaps, accuracy of this model.

$\mathrm{T}$ he susceptibility of ferrets to influenza virus infection has been known for nearly a century. Ferrets and humans share similarities in lung physiology, cellular receptor distribution, and clinical signs of infection, making the ferret an attractive small mammalian model for laboratory study of influenza viruses (1). Influenza virus infection in ferrets emulates the severe disease elicited by highly pathogenic avian influenza viruses in humans and the transmissibility of seasonal human influenza viruses via respiratory

Author affiliations: Centers for Disease Control and Prevention, Atlanta, Georgia, USA (J.A. Belser); Imperial College, London, UK (W. Barclay); Doherty Institute, Melbourne, Victoria, Australia (I. Barr, K. Subbarao); Erasmus Medical Center, Rotterdam, the Netherlands (R.A.M. Fouchier); Hokkaido University, Sapporo, Japan (R. Matsuyama, H. Nishiura); The University of Hong Kong, Hong Kong, China (M. Peiris, H. Zhu, H.-L. Yen); St. Jude Children's Research Hospital, Memphis, Tennessee, USA (C.J. Russell)

DOI: https://doi.org/10.3201/eid2406.172114 droplets. Commonly reported experimental setups for the study of influenza virus transmission in ferrets include cohousing influenza virus-infected and uninfected ferrets (previously termed the direct contact model) or physically separating virus-infected and uninfected ferrets (previously termed the respiratory droplet model or the airborne transmission model) $(1,2)$. In the co-housing design, transmission between ferrets can be mediated by any of the multiple routes that facilitate influenza virus transmission, including direct contact, indirect contact via fomites, or via respiratory droplets (airborne particles with $>5 \mu \mathrm{m}$ aerodynamic diameter) and droplet nuclei (airborne particles with $<5 \mu \mathrm{m}$ aerodynamic diameter). In the physical separation design, direct or indirect contact between donor and recipient ferrets is precluded by separating cages with a side panel or cage walls that permit air exchange between cages but prevent direct contact between ferrets. If the recipient ferret becomes infected with influenza virus, respiratory droplets and droplet nuclei expelled from the donor ferret represent the only possible source of transmission. Many influenza viruses that cause zoonotic infections in humans (e.g., most swine influenza viruses, avian influenza viruses of subtype H5N1 and other subtypes) are generally poorly transmitted between ferrets via respiratory droplets and droplet nuclei, whereas viruses associated with seasonal epidemics or pandemics in humans (e.g., influenza A[H1N1]pdm09 virus, the reconstructed 1918 virus) can be transmitted relatively efficiently (3-7). As such, the ferret model provides a useful tool for research on influenza virus transmission and pandemic risk assessment. Influenza transmissibility between ferrets is a parameter included in tools for assessing the potential pandemic risk for zoonotic influenza viruses: the Influenza Risk Assessment Tool (8) and the World Health Organization Tool for Influenza Pandemic Risk Assessment (9).

Recent experimental studies performed by using ferret transmission models have greatly expanded knowledge of influenza virus transmissibility. Among other findings, these studies have identified molecular correlates and determinants of airborne virus spread, differential innate host 
responses between viruses with distinct transmissible phenotypes, and the role of vaccination and antiviral administration in mitigating virus spread to susceptible contacts $(2,10-19)$. Although the ferret model does not always recapitulate virus transmissibility observed among humans, possibly because of differences in prior exposure history and other unidentified host factors, these studies, in isolation and in tandem with additional laboratory experiments, have improved our ability to perform informative risk assessments of novel and emerging influenza viruses. For example, the limited transmissibility of influenza A(H7N9) viruses via airborne particles in ferrets, in the absence of sustained human-to-human transmission, indicates that the pandemic threat posed by this virus subtype is relatively higher than that of other studied avian influenza viruses; further refinements to the experimental model may help explain why this virus does not currently spread readily between humans.

Despite the growing use of influenza virus transmission studies in the field, there is a wide and often underappreciated heterogeneity among these studies with regard to assessing influenza virus transmissibility via airborne particles in the ferret model. This heterogeneity includes host-specific, virus-specific, and environmental/laboratoryspecific variables (Table 1) that are a help and a hindrance for understanding the relative risk for virus transmission with a particular virus strain or subtype (1). Robust data can be generated by several independent research groups performing parallel studies with genetically similar but not identical field viruses. Such an approach reduces potential strain-specific or method-specific biases. Conversely, each experimental variable may restrict or complicate direct

Table 1. Examples of heterogeneity in experimental designs among published risk-assessment studies using ferrets as models for influenza virus transmission studies and pandemic risk assessments*

\begin{tabular}{lc}
\hline Parameter & Examples of variability \\
\hline Virus (before ferret & Seed stock passage history, stock \\
introduction) & $\begin{array}{c}\text { growth matrix, stock titer, wild-type vs. } \\
\text { reverse genetics, plaque-purified vs. } \\
\text { quasispecies, storage and propagation }\end{array}$
\end{tabular}

\begin{tabular}{lc}
\hline $\begin{array}{l}\text { Ferret (before virus } \\
\text { introduction) }\end{array}$ & $\begin{array}{c}\text { Source/genetic lineage, serostatus, age, } \\
\text { sex, weight, neutered or intact status, } \\
\text { hormonal treatment (females), anesthetic } \\
\text { used, housing conditions }\end{array}$ \\
\hline Virus inoculation & $\begin{array}{c}\text { Inoculation route, method, dose, and } \\
\text { volume; buffer for dilution }\end{array}$ \\
\hline $\begin{array}{l}\text { Transmission } \\
\text { experimental designs }\end{array}$ & $\begin{array}{c}\text { Donor:recipient ratio, number of } \\
\text { replicates per containment, caging size } \\
\text { and setup, perforation size and exposure } \\
\text { area between cages, distance between } \\
\text { cages, directional airflow, air changes } \\
\text { per hour, temperature and humidity, } \\
\text { timing and duration of exposure, } \\
\text { frequency and sites for sample collection }\end{array}$ \\
\hline *References for individual studies using these conditions are described \\
in (1).
\end{tabular}

comparisons of data from different research groups or institutions, depending on each variable's effect on the transmission outcome, making it impossible to combine data.

Current knowledge about viral, host, and environmental factors that may drive transmission is limited. To facilitate interpretation of data generated in different laboratories, efforts should be made to improve transparency in descriptions of methods and to better differentiate what constitutes a risk-assessment activity versus a research activity. After the Transmission of Respiratory Viruses conference, held June 19-21, 2017, in Hong Kong (20), an ancillary workshop was held on June 22, 2017, to discuss this topic.

Our article serves as a starting point for highlighting potential heterogeneity in ferret transmission experimental designs for future refinement. It is not intended as a policy statement for detailed recommendations on ferret experimental designs, when the effects of many of these variables are not fully understood. We discuss and summarize variables in the ferret transmission model.

\section{Identifying Variability}

Major drivers of variability in studies of influenza virus transmissibility include differences in virus strain, dose, and inoculation route (21-23). Even when a common virus strain is used, results are potentially affected by the passage condition (e.g., multiplicity of infection), passage history (in embryonated chicken eggs or in mammalian cells), and storage condition of the virus. Risk-assessment studies are particularly challenging because the evaluations are conducted with recently isolated specimens that are not available from a central respository before animal inoculation; variation pertaining to input stock material is possible even when different laboratories have confirmed sequence identity of the same virus undergoing evaluation. Furthermore, several other factors have not been directly demonstrated but are likely to contribute to experimental variation in transmission studies: cage design, air flow direction, air changes per hour, facility-specific temperature and humidity levels, and others (Table 1) (1). Beyond these, additional variables, which are largely out of the control of researchers, include ferret suppliers (commercial or hobby and the quantity of ferrets available from each), countryspecific animal welfare issues, institutional animal care and use committee guidelines, and pharmaceutical limitations (availability or restriction of anesthetics licensed for use).

Although some factors are considered to be controllable, much of the variability between groups cannot be easily overcome. For example, the absence of commercially available uniform caging for ferret transmission experiments (a reflection of country- and institution-specific size regulations and facilities constraints) represents a parameter that would be difficult to standardize. However, 
a greater understanding of the relative role and contribution of different variables can improve our ability to better contextualize and interpret results among laboratories. For example, does the virus dose affect transmissibility or otherwise influence detection of transmissible quasispecies in contact ferrets? Gustin et al. previously demonstrated the potential effects of the dose and the route of inoculation (intranasal vs. aerosol) on transmission potential (22), suggesting the need to standardize these 2 parameters for riskassessment studies. Similarly, the use of directional airflow or air changes per hour in ferret housing apparatuses is not always specified in reports of risk-assessment results. Anecdotally, these variables seem to play a role in modulation of virus transmissibility, and they should be examined systematically to ascertain which parameters (including but not limited to those shown in Table 1) would benefit from standardization, where possible, across laboratories in the field. This standardization and interpretation can take place only when all known major drivers of laboratory variability that influence virus transmissibility are described along with the results.

As discussed at the workshop, the participating laboratories analyzed the protocols used for evaluation of influenza virus transmissibility via airborne particles in the ferret model, which highlighted the breadth of experimental designs. It was also clear that all variables that are probable contributors to differential results are not routinely disclosed in peer-reviewed publications or other platforms where results are discussed. Lack of disclosure of all variables can complicate the ease of comparing findings between laboratories, warranting a closer look at the feasibility of using a more comparable study design between multiple laboratory groups for risk-assessment studies. As such, a push toward comprehensive description of specific experimental conditions would aid this effort and would probably be valuable when risk assessments performed in different laboratories are compared.

\section{Defining Risk Assessment}

Beyond the experiment- and facility-based variability we describe, the lack of a standard protocol for the number of experimentally infected animals and the number of recipient ferrets (donor:recipient ratios) included in ferret transmission studies can affect the ability to interpret results among groups and represents a substantial controllable parameter. The ideal standard for risk assessment activities seems to be a 1:1 donor:recipient experimental setup to assess virus transmissibility via the airborne route, where each virus-infected ferret is exposed to only 1 recipient ferret. This design facilitates ease of interpretation of results and provides added rigor from a statistical perspective. This design also restricts exposure to virus-laden particles expelled from each donor ferret to its respective recipient, ensuring that any detected transmission event would have originated from exposure to separate donors. However, because of space limitations, these experiments are often conducted in replicates inside a single physical containment area with a shared ventilation system (i.e., housing multiple pairs of donors and respiratory droplet recipients in separate cages with shared air) while still maintaining a 1:1 donor:recipient ratio. If transmission is mediated by virusladen particles expelled by donors, increasing the number of donors within a single containment area is likely to increase the concentration of virus-laden particles in the air, thereby increasing the observed transmissibility. Specifically, it is not known whether transmission kinetics would be comparable if 3 independent experiments were performed with 1 donor to 1 recipient (each ferret housed singly) versus 1 experiment with 3 donors and 3 recipients per containment area. Air sampling devices that allow monitoring of the quantities of virus-laden particles in the air throughout the experiment would help refine the experiment outcomes and are likely to become part of these experimental designs in the future.

Further impeding efforts to compare results among laboratories, many experiments include an additional contact ferret co-housed with an experimentally infected ferret to evaluate virus transmissibility in a direct contact setting while still assessing the respiratory droplet transmission to a recipient ferret housed in an adjacent cage. In this design, several ferrets may serve as donors (i.e., virus-inoculated ferrets, co-housed ferrets that became infected as a result of direct/indirect contact, or both). Moreover, the donor and direct contact-infected ferrets are likely to shed virusladen particles at different times, further complicating the results of the transmission experiments. Ideally, the effects of different experimental designs should be investigated in systematic experiments, and researchers should strive to disclose this information as comprehensively as possible.

During workshop discussions, most researchers agreed that it would be helpful for the field to coalesce around a fixed 1:1 donor:recipient ratio (with or without several discrete pairs inside 1 physical containment area) for risk-assessment transmission experiments. Introduction of direct contact ferrets into the experimental setup would probably extend the amount of time that virus-laden particles can be released in the air. Virus amplification by direct contact ferrets may also lead to virus adaptation and emergence of variants with increased respiratory droplet transmission potential. Applying a 1:1 donor:recipient ratio would increase the consistency of the experimental design under which risk-assessment experiments are conducted across multiple laboratories, differentiating them from broader, more heterogeneous research-based assessments that would include more experimental designs and variables. However, individual laboratories have built up datasets and experience 
over the years while performing risk-assessment studies with different strains of influenza viruses; thus, adopting a common protocol may be difficult to achieve within a short time.

Although viruses that are readily transmitted by the airborne route will exhibit robust transmission in a direct contact setting, some influenza viruses that are not transmitted efficiently via respiratory droplets are nonetheless transmitted between ferrets placed in direct contact, which facilitates transmission via multiple modes. Studies evaluating virus transmissibility between ferrets placed in direct contact may be influenced by many of the experimental drivers discussed here; when using this model, further contributions to variability are introduced by animal behavior and housing practices. Although scoring for the Centers for Disease Control and Prevention Influenza Risk Assessment Tool includes data derived from the direct contact transmission model in risk assessment, it is not fully clear how to interpret the relative pandemic risk resulting from viruses that transmit in a direct contact setting but not via respiratory droplets. As discussed above, a greater understanding of what confers virus transmissibility in both models will improve our ability to interpret results from more permissive direct contact models with the more stringent respiratory droplet transmissibility. This knowledge will improve our ability to appropriately include and aggregate results from both types of transmission studies in influenza virus risk assessments.

\section{Limitations of the Ferret Transmission Model}

Although the ferret transmission model has greatly improved our understanding of influenza virus transmissibility, there are limits to what this model can contribute to risk assessment and how results are interpreted. In particular, inefficient virus transmission (e.g., when 1 of 3 recipient ferrets becomes productively infected) remains a difficult outcome to understand. It is often unclear whether this event results from genetic changes in the virus during the transmission event, reflects the transmitted infectious dose, or results from other contributing factors; concurrent contextualization of these results with other laboratory paramaters (inclusive of in vivo, in vitro, and aerobiology-based experimentation) can often provide additional insight. Similarly, interpretation of seroconversion in the absence of detectable virus in respiratory secretions or detectable virus in the absence of seroconversion can be difficult. Moving toward a consensus on the implications of inefficient transmission events would be helpful because, currently, efficient and inefficient transmission are not well defined.

Another major limitation of current ferret transmission studies is the small group size, which is driven by cost, size of the animals and their associated housing requirements, and ethical and practical constraints. For this reason, statistical analyses of data from transmission experiments are infrequently performed $(24,25)$, and repetition of positive-control viruses is not uniformly feasible. Risk assessment studies are often performed with 3-4 replicates of transmission pairs. With this sample size, it is feasible to statistically infer virus transmissibility at the extremes of transmission potential (i.e., virus transmission to 4 of 4 ferrets versus 0 of 4 ferret pairs), but statistical power to compare viruses with intermediate transmissibility (transmissibility to 2 or 3 of 4 pairs) is limited. The opportunity for meta-analyses that combine results from different laboratories could be beneficial, especially for monitoring minor changes in transmission potential of a particular zoonotic virus as it evolves over time. However, meta-analyses can be performed only when experiments use comparable study designs, especially with regard to those parameters known to most dramatically influence virus transmissibility.

\section{Potential Refinements of the Ferret Transmission Model}

Great efforts are being made to reduce the limitations discussed above by using novel and emerging technologies and research-based approaches. For identifying mutations that may have occurred during transmission events, Sanger sequencing has been frequently used. Recently developed technologies (e.g., use of neutral barcodes to individually track influenza viruses in a population) or deep-sequencing approaches have provided, and probably will continue to provide, additional information to aid in the interpretation of inefficient virus transmission events, elucidate transmission bottlenecks, and differentiate between within-group variability and larger differences in experimental setup and design (26-28). Although incorporating viral genome sequencing in all risk-assessment studies would be beneficial, the inclusion and standardization of these approaches represents a substantial challenge with regard to sample choice for testing (types of samples, dates of sample collection, titers of samples) and institutional restrictions on collection, interpretation, and dissemination of this information.

An additional avenue for improved understanding of virus transmissibility via respiratory droplets are aerobiology-based approaches. These approaches include analysis of the exhaled breath of infected ferrets and the amount and size distribution of virus-containing aerosols released by infected ferrets (29-31). Although it is unlikely that aerobiology-based information can be incorporated into standard risk-assessment ferret experiments, information gained from these experiments could improve our understanding and interpretation of influenza virus transmissibility in the ferret model, providing additional 
data about the contributions of different variables to consistency between laboratories for experiments assessing virus transmission.

In vivo ferret transmission studies are not performed in isolation. The incorporation of these data into larger research efforts has greatly expanded our understanding of the complex determinants of influenza virus transmission in mammals. For example, hemagglutinin acid stability and the hemagglutinin-neuraminidase balance have been linked with virus transmissibility via the airborne route in ferrets, as have receptor binding preference, gene constellation, neuraminidase stalk length, and other parameters $(14-17,28,29)$. In addition, studies examining the relative effects of environmental temperature and relative humidity on influenza virus stability and transmissibility (underscoring the need to report this information more specifically in published methods sections) will provide needed information pertaining to the seasonality of influenza virus spread in humans $(32,33)$. Further refinement of the ferret model concurrent with studies using other modeling approaches, including but not limited to in vitro and ex vivo infection models, will continue to support in vivo transmission risk assessments in this species.

\section{Moving Forward}

The plasticity of the ferret model permits a wide range of experimental approaches to assess influenza virus transmissibility. This plasticity represents a great advantage when designing research experiments to evaluate viral, host, environmental, and other factors that contribute to transmission between mammals. However, it might be beneficial for studies conducted primarily for risk-assessment purposes to be performed under conditions as uniform as possible. For example, moving toward a standardized 1:1 donor:recipient ratio in risk-assessment studies would probably enhance the comparability of results found by different research groups and would enable inclusion in metaanalyses (Table 2).

Current knowledge regarding the viral, host, and environmental parameters that drive transmission outcomes is limited. Understanding these parameters would be beneficial for infection control, and future studies should aim to validate these factors empirically. As data regarding the exact role of each of the potential parameters discussed in this article are developed, improved documentation of variables (Table 1) associated with risk assessments would facilitate comparison of data generated across different

Table 2. Features that may be conducive to uniform, reproducible risk-assessment transmission setups when using ferrets as models for influenza virus transmission studies and pandemic risk assessments*

\begin{tabular}{|c|c|c|c|}
\hline Property & Rationale & Sample phrasing & $\begin{array}{l}\text { Perceived } \\
\text { importance }\end{array}$ \\
\hline Donor:recipient ratio of $1: 1$ & $\begin{array}{l}\text { Improved statistical rigor, potential } \\
\text { application for meta-analysis, and } \\
\text { interpretation of results. The number of } \\
\text { donor:recipient pairs housed inside } \\
\text { containment with shared ventilation } \\
\text { should be reported. }\end{array}$ & $\begin{array}{l}\text { "Inoculated ferrets }(n=3) \text { were each placed in } \\
\text { a separate cage; } 24 \text { hours later, naïve ferrets } \\
(n=3) \text { were each placed in a different cage } \\
\text { adjacent to an inoculated ferret." }\end{array}$ & High \\
\hline Seronegative ferrets & $\begin{array}{l}\text { Prior influenza virus exposure history } \\
\text { can be difficult to quantify and control. } \\
\text { The methods used for assessing prior } \\
\text { exposure should be disclosed. }\end{array}$ & $\begin{array}{c}\text { "Ferrets were serologically negative to currently } \\
\text { circulating influenza A ( } \mathrm{H} 1 \mathrm{~N} 1 \text { and } \mathrm{H} 3 \mathrm{~N} 2) \text { and } \mathrm{B} \\
\text { viruses before challenge, as confirmed by } \mathrm{HI} \\
\text { assay." }\end{array}$ & High \\
\hline $\begin{array}{l}\text { Harmonization of } \\
\text { ventilation and } \\
\text { environmental conditions }\end{array}$ & $\begin{array}{l}\mathrm{ACH} \text {, directional airflow, cage design, } \\
\text { humidity/temperature information are } \\
\text { reported concurrent with release of } \\
\text { results. }\end{array}$ & $\begin{array}{l}\text { "Ferrets were housed for the duration of the } \\
\text { experiment in an environmental chamber with } \\
\text { HEPA filtration operating at } 20 \mathrm{ACH} \text {. Airflow } \\
\text { velocity was found to be negligible between } \\
\text { donor and recipient cages. Ambient } \\
\text { temperature }\left(20^{\circ}-22^{\circ} \mathrm{C}\right) \text { and relative humidity } \\
(40 \%) \text { were monitored during the experiment." }\end{array}$ & High \\
\hline $\begin{array}{l}\text { Uniform definition of } \\
\text { efficient transmissibility }\end{array}$ & $\begin{array}{c}\text { Virus titers (with detection limit) and } \\
\text { seroconversion are both required to } \\
\text { demonstrate robust transmission } \\
\text { event. }\end{array}$ & $\begin{array}{l}\text { "Virus transmissibility was confirmed by } \\
\text { detection of infectious virus and by } \\
\text { seroconversion to homologous virus in recipient } \\
\text { ferrets." }\end{array}$ & High \\
\hline $\begin{array}{l}\text { Dose, volume, and route of } \\
\text { inoculation }\end{array}$ & $\begin{array}{c}\text { Dose of inoculum may affect the } \\
\text { transmission kinetics (22). A } \\
\text { consensus within the risk-assessment } \\
\text { group may be beneficial. }\end{array}$ & $\begin{array}{l}\text { "Ferrets were inoculated by the intranasal route } \\
\text { with } 10^{6} \mathrm{PFU} \text { of virus in a volume of } 500 \mu \mathrm{L} \text { " }\end{array}$ & High \\
\hline $\begin{array}{l}\text { Application of air sampling } \\
\text { device to determine the } \\
\text { size and quantity of virus- } \\
\text { laden particles in air }\end{array}$ & $\begin{array}{l}\text { The results may help correlate and } \\
\text { refine the transmission phenotype. }\end{array}$ & $\begin{array}{c}\text { "Variables were inclusive of vendor, duration of } \\
\text { sampling, specification of collection matrices } \\
\text { (buffers, gelatin[s], etc.), specification of virus } \\
\text { confirmation via PCR and/or live virus } \\
\text { detection, normalization correction of data (if } \\
\text { applicable)." }\end{array}$ & Intermediate \\
\hline
\end{tabular}

(20). ACH, air changes per hour; HEPA, high-efficiency particulate air. 
laboratories. These should include, but are not limited to, stating the stock passage history and storage conditions; dose, volume, and route of inoculation; donor:recipient ratio (and the number of donor:recipient pairs present within a single containment area); and clarity in describing cage setup (coupled with illustrations when possible for easy understanding of the overall experimental conditions), specifying room and cage humidity/temperature, stating airflow directionality when present and air changes per hour, and specifying the condition of sample storage and processing procedures (e.g., whether samples are titered immediately for presence of infectious virus, or whether they are frozen and thawed before use).

Many unanswered questions are relevant for understanding the pandemic risk posed by novel and emerging influenza viruses that lie outside a standardized risk-assessment experimental setup. Some studies that would provide additional valuable information include modulation of distance between cages when assessing virus transmissibility via the airborne route, shortening the duration of exposure between inoculated donors and contact ferrets $(18,28,34)$, and modifying the donor:recipient ferret ratio. Performing these types of experiments according to a standardized risk-assessment evaluation of virus transmissibility could provide valuable contextual information for a more nuanced risk assessment. Distinguishing between risk assessment and research activities will greatly facilitate interpretation and contextualization of data generated by different laboratories.

A useful exercise might be for several research groups to test and compare results of a transmission experiment by using 1 selected virus strain prepared by 1 laboratory. Many practical considerations would need to be discussed, including the particular strain to test and the anticipated transmission phenotype of this virus. Complete standardization of ferret transmission studies conducted worldwide is not possible; however, exercises that seek to examine the relative contributions of laboratory-specific drivers of experimental variability may identify critical parameters or conditions. Similarly, meta-analyses of published data, with included disclosure of the parameters listed in Table 1, could aid in our understanding of the relative contribution of variables involved in respiratory droplet transmission experimentation in ferrets.

Influenza viruses will continue to jump the species barrier and cause human infection and disease. Virustransmissibility assessments in the ferret model represent one of numerous activities conducted to aid pandemic preparedness efforts in the event that a pandemic virus does emerge in the future. Continued refinement of the ferret model, concurrent with advances in identification of viral, host, and environmental factors that influence transmission risk (35), will facilitate assessments of novel and emerging influenza viruses and aid development of better infection control measures.
The workshop was supported by the Theme-based Research Scheme (project no. T11-705/14N) from the Government of Hong Kong Special Administrative Region, China.

\section{About the Author}

Dr. Belser is a microbiologist at the Centers for Disease Control and Prevention, Atlanta, Georgia. Her research interests include the pathogenicity, transmissibility, and tropism of influenza viruses, including $\mathrm{H} 7$ subtype viruses with pandemic potential.

\section{References}

1. Belser JA, Eckert AM, Tumpey TM, Maines TR. Complexities in ferret influenza virus pathogenesis and transmission models. Microbiol Mol Biol Rev. 2016;80:733-44. http://dx.doi.org/ 10.1128/MMBR.00022-16

2. Herfst S, Schrauwen EJ, Linster M, Chutinimitkul S, de Wit E, Munster VJ, et al. Airborne transmission of influenza A/H5N1 virus between ferrets. Science. 2012;336:1534-41. http://dx.doi.org/10.1126/science. 1213362

3. Zhu H, Wang D, Kelvin DJ, Li L, Zheng Z, Yoon SW, et al. Infectivity, transmission, and pathology of human-isolated H7N9 influenza virus in ferrets and pigs. Science. 2013;341:183-6. http://dx.doi.org/10.1126/science.1239844

4. Tumpey TM, Maines TR, Van Hoeven N, Glaser L, Solórzano A, Pappas C, et al. A two-amino acid change in the hemagglutinin of the 1918 influenza virus abolishes transmission. Science. 2007;315:655-9. http://dx.doi.org/10.1126/science. 1136212

5. Yen HL, Lipatov AS, Ilyushina NA, Govorkova EA, Franks J, Yilmaz N, et al. Inefficient transmission of H5N1 influenza viruses in a ferret contact model. J Virol. 2007;81:6890-8. http://dx.doi.org/10.1128/JVI.00170-07

6. Maines TR, Chen LM, Matsuoka Y, Chen H, Rowe T, Ortin J, et al. Lack of transmission of H5N1 avian-human reassortant influenza viruses in a ferret model. Proc Natl Acad Sci U S A. 2006;103:12121-6. http://dx.doi.org/10.1073/pnas.0605134103

7. Munster VJ, de Wit E, van den Brand JM, Herfst S, Schrauwen EJ, Bestebroer TM, et al. Pathogenesis and transmission of swineorigin $2009 \mathrm{~A}(\mathrm{H} 1 \mathrm{~N} 1)$ influenza virus in ferrets. Science. 2009;325:481-3.

8. Cox NJ, Trock SC, Burke SA. Pandemic preparedness and the Influenza Risk Assessment Tool (IRAT). Curr Top Microbiol Immunol. 2014;385:119-36. http://dx.doi.org/10.1007/82_2014_419

9. World Health Organization. Tool for Influenza Pandemic Risk Assessment (TIPRA). Geneva: The Organization; 2016.

10. Imai M, Watanabe T, Hatta M, Das SC, Ozawa M, Shinya K, et al. Experimental adaptation of an influenza H5 HA confers respiratory droplet transmission to a reassortant $\mathrm{H} 5 \mathrm{HA} / \mathrm{H} 1 \mathrm{~N} 1$ virus in ferrets. Nature. 2012;486:420-8.

11. Maines TR, Belser JA, Gustin KM, van Hoeven N, Zeng H, Svitek N, et al. Local innate immune responses and influenza virus transmission and virulence in ferrets. J Infect Dis. 2012; 205:474-85. http://dx.doi.org/10.1093/infdis/jir768

12. Oh DY, Lowther S, McCaw JM, Sullivan SG, Leang SK, Haining J, et al. Evaluation of oseltamivir prophylaxis regimens for reducing influenza virus infection, transmission and disease severity in a ferret model of household contact. J Antimicrob Chemother. 2014;69:2458-69. http://dx.doi.org/10.1093/jac/dku146

13. Houser KV, Pearce MB, Katz JM, Tumpey TM. Impact of prior seasonal H3N2 influenza vaccination or infection on protection and transmission of emerging variants of influenza $\mathrm{A}(\mathrm{H} 3 \mathrm{~N} 2) \mathrm{v}$ virus in ferrets. J Virol. 2013;87:13480-9. http://dx.doi.org/10.1128/ JVI.02434-13 
14. Linster M, van Boheemen S, de Graaf M, Schrauwen EJA, Lexmond P, Mänz B, et al. Identification, characterization, and natural selection of mutations driving airborne transmission of A/H5N1 virus. Cell. 2014;157:329-39. http://dx.doi.org/10.1016/ j.cell.2014.02.040

15. Russier M, Yang G, Rehg JE, Wong SS, Mostafa HH, Fabrizio TP, et al. Molecular requirements for a pandemic influenza virus: an acid-stable hemagglutinin protein. Proc Natl Acad Sci U S A. 2016;113:1636-41. http://dx.doi.org/10.1073/pnas.1524384113

16. Yen HL, Liang CH, Wu CY, Forrest HL, Ferguson A, Choy KT, et al. Hemagglutinin-neuraminidase balance confers respiratorydroplet transmissibility of the pandemic H1N1 influenza virus in ferrets. Proc Natl Acad Sci U S A. 2011;108:14264-9. http://dx.doi.org/10.1073/pnas.1111000108

17. Blumenkrantz D, Roberts KL, Shelton H, Lycett S, Barclay WS. The short stalk length of highly pathogenic avian influenza H5N1 virus neuraminidase limits transmission of pandemic H1N1 virus in ferrets. J Virol. 2013;87:10539-51. http://dx.doi.org/10.1128/ JVI.00967-13

18. Paules CI, Lakdawala S, McAuliffe JM, Paskel M, Vogel L, Kallewaard NL, et al. The hemagglutinin A stem antibody MEDI8852 prevents and controls disease and limits transmission of pandemic influenza viruses. J Infect Dis. 2017;216:356-65. http://dx.doi.org/10.1093/infdis/jix292

19. Baz M, Boonnak K, Paskel M, Santos C, Powell T, Townsend A, et al. Nonreplicating influenza A virus vaccines confer broad protection against lethal challenge. MBio. 2015;6:e01487-15. http://dx.doi.org/10.1128/mBio.01487-15

20. Cowling BJ, Lam TT-Y, Yen HL, Poon LLM, Peiris M. Evidencebased options for controlling respiratory virus transmission. Emerg Infect Dis. 2017 Nov [cited 2018 Feb 20]. http://dx.doi.org/ 10.3201/eid2311.171231

21. Moore IN, Lamirande EW, Paskel M, Donahue D, Kenney H, Qin J, et al. Severity of clinical disease and pathology in ferrets experimentally infected with influenza viruses is influenced by inoculum volume. J Virol. 2014;88:13879-91. http://dx.doi.org/ 10.1128/JVI.02341-14

22. Gustin KM, Belser JA, Wadford DA, Pearce MB, Katz JM, Tumpey TM, et al. Influenza virus aerosol exposure and analytical system for ferrets. Proc Natl Acad Sci U S A. 2011;108:8432-7. http://dx.doi.org/10.1073/pnas.1100768108

23. Belser JA, Gustin KM, Maines TR, Pantin-Jackwood MJ, Katz JM, Tumpey TM. Influenza virus respiratory infection and transmission following ocular inoculation in ferrets. PLoS Pathog. 2012; 8:e1002569. http://dx.doi.org/10.1371/journal.ppat.1002569

24. Nishiura H, Yen HL, Cowling BJ. Sample size considerations for one-to-one animal transmission studies of the influenza A viruses. PLoS One. 2013;8:e55358. http://dx.doi.org/10.1371/ journal.pone. 0055358

25. Belser JA, Maines TR, Katz JM, Tumpey TM. Considerations regarding appropriate sample size for conducting ferret transmission experiments. Future Microbiol. 2013;8:961-5. http://dx.doi.org/10.2217/fmb.13.64
26. Frise R, Bradley K, van Doremalen N, Galiano M, Elderfield RA, Stilwell P, et al. Contact transmission of influenza virus between ferrets imposes a looser bottleneck than respiratory droplet transmission allowing propagation of antiviral resistance. Sci Rep. 2016;6:29793. http://dx.doi.org/10.1038/ srep29793

27. Varble A, Albrecht RA, Backes S, Crumiller M, Bouvier NM, Sachs D, et al. Influenza A virus transmission bottlenecks are defined by infection route and recipient host. Cell Host Microbe. 2014;16:691-700. http://dx.doi.org/10.1016/ j.chom.2014.09.020

28. Lakdawala SS, Jayaraman A, Halpin RA, Lamirande EW, Shih AR, Stockwell TB, et al. The soft palate is an important site of adaptation for transmissible influenza viruses. Nature. 2015;526:122-5. http://dx.doi.org/10.1038/nature15379

29. Lakdawala SS, Lamirande EW, Suguitan AL Jr, Wang W, Santos CP, Vogel L, et al. Eurasian-origin gene segments contribute to the transmissibility, aerosol release, and morphology of the 2009 pandemic H1N1 influenza virus. PLoS Pathog. 2011;7:e1002443. http://dx.doi.org/10.1371/journal.ppat.1002443

30. Gustin KM, Katz JM, Tumpey TM, Maines TR. Comparison of the levels of infectious virus in respirable aerosols exhaled by ferrets infected with influenza viruses exhibiting diverse transmissibility phenotypes. J Virol. 2013;87:7864-73. http://dx.doi.org/10.1128/ JVI.00719-13

31. Koster F, Gouveia K, Zhou Y, Lowery K, Russell R, MacInnes H, et al. Exhaled aerosol transmission of pandemic and seasonal H1N1 influenza viruses in the ferret. PLoS One. 2012;7:e33118. http://dx.doi.org/10.1371/journal.pone.0033118

32. Lowen AC, Steel J. Roles of humidity and temperature in shaping influenza seasonality. J Virol. 2014;88:7692-5. http://dx.doi.org/10.1128/JVI.03544-13

33. Gustin KM, Belser JA, Veguilla V, Zeng H, Katz JM, Tumpey TM, et al. Environmental conditions affect exhalation of $\mathrm{H} 3 \mathrm{~N} 2$ seasonal and variant influenza viruses and respiratory droplet transmission in ferrets. PLoS One. 2015;10:e0125874. http://dx.doi.org/10.1371/ journal.pone. 0125874

34. Roberts KL, Shelton H, Stilwell P, Barclay WS. Transmission of a $2009 \mathrm{H} 1 \mathrm{~N} 1$ pandemic influenza virus occurs before fever is detected, in the ferret model. PLoS One. 2012;7:e43303. http://dx.doi.org/10.1371/journal.pone.0043303

35. Lipsitch M, Barclay W, Raman R, Russell CJ, Belser JA, Cobey S, et al. Viral factors in influenza pandemic risk assessment. eLife. 2016;5:5. http://dx.doi.org/10.7554/eLife.18491

Address for correspondence: Jessica A. Belser, Centers for Disease Control and Prevention, 1600 Clifton Rd NE, Mailstop G16, Atlanta, GA 30329-4027, USA; email: jax6@cdc.gov; Hui-Ling Yen, The University of Hong Kong-School of Public Health, 6F, Laboratory Block, LKS Faculty of Medicine, No. 21 Sassoon Rd, Hong Kong SAR, China; email: hyen@hku.hk 